\title{
Usabilidad y prospectiva del aprendizaje a distancia en Formación Profesional determinado por la competencia digital
}

Antonio-José Moreno-Guerrero

Universidad de Granada, España

Email: ajmorenodugr.es

ORCID: 0000-0003-3191-2048

\section{Santiago Pozo-Sánchez}

Universidad de Granada, España

Email: santiagopozodcorreo.ugr.es

ORCID: 0000-0001-8125-4990
Jesús López-Belmonte

Universidad de Granada, España

Email: jesuslopezaugr.es

ORCID: 0000-0003-0823-3370

Juan-Antonio López-Núñez

Universidad de Granada, España

Email: juanlopedugr.es

ORCID: 0000-0001-9881-9169

\section{RESUMEN}

Las tecnologías de la información y comunicación han entrado de lleno en la sociedad y, por ende, en la Formación Profesional. El objetivo del estudio es identificar y predecir la incidencia de la competencia digital en el uso del aprendizaje en línea en el profesorado de Formación Profesional. El método de investigación es de tipo descriptivo, correlacional y predictivo, en una muestra de 627 docentes de la geografía española. Los datos han sido recopilados por medio de un cuestionario. Los resultados muestran un déficit competencial en diversas áreas de la competencia digital, algo que afecta directamente en la aplicación del aprendizaje en línea, aunque los niveles en las áreas de información y alfabetización digital, en la resolución de problemas y en las destrezas de comunicación y colaboración pueden marcar el uso del método e-learning por parte del profesorado.

Palabras clave: Innovación educacional, aprendizaje en línea, competencia digital, formación profesional.

\section{Usability and prospective of distance learning in Vocational Training determined by digital competence}

\begin{abstract}
Information and communication technologies have fully entered society and, therefore, vocational training. The objective of the study is to identify and predict the incidence of digital competence in the use of online learning in Vocational Training teachers. The research method is descriptive, correlational and predictive, in a sample of 627 teachers from the Spanish geography. The data has been collected by means of a questionnaire. The results show a competence deficit in various areas of digital competence, something that directly affects the application of online learning, although the levels in the areas of information and digital literacy, problem solving and communication skills and Collaboration can mark the use of the e-learning method by teachers.
\end{abstract}

Key words: Educational innovations, online learning, digital competence, vocational training. 


\section{Introducción}

La expansión de las tecnologías de la información y comunicación (TIC) en todos los sectores de la sociedad es ya una realidad, especialmente en el sector de la educación, donde los procesos de enseñanza y aprendizaje de todas las etapas educativas - ya sean formales o no formales - hacen cada vez un mayor uso de las mismas (Larionova, Brown, Bystrova y Sinitsyn, 2018), dando origen a una educación propia de una era tecnológica (Viñals y Cuenca, 2016). En consecuencia, los nuevos paradigmas educacionales de corte innovador suponen una serie de requerimientos en el profesorado (Area, 2015), debiendo estos disponer de una formación pertinente en el uso de las TIC, así como de un nivel de competencia digital adecuado (López, Pozo, Fuentes y Romero, 2019) para poder desarrollar metodologías activas e innovadoras en la acción formativa (Elche y Yubero, 2019) y dar paso a la integración de todo tipo de equipamientos tecnológicos en los centros educativos (Area, Hernández y Sosa, 2016).

En este sentido, para que la proyección de las TIC en los procesos formativos sea eficaz es conveniente que los docentes dispongan de la capacidad de utilizar diversos dispositivos tecnológicos, así como otros condicionantes tales como el apoyo por parte de la propia administración educativa, la percepción de sensaciones positivas hacia su utilización y - finalmente- obtener resultados satisfactorios al final del proceso de enseñanza y aprendizaje (Moreira, García, Conde y González, 2019).

Todo ello conlleva que la educación esté encaminada al desarrollo de diversas competencias en los propios estudiantes, pero antes, es necesario que los docentes las tengan adquiridas (Rodríguez, Romero y Agreda, 2019), como ya estableció el Parlamento Europeo y el Consejo sobre competencias claves para el aprendizaje permanente celebrado en el año 2006. Entre todas las competencias clave que establecen se encuentra la competencia digital, concebida como un concepto dinámico que va cambiando y actualizándose al mismo ritmo que los avances tecnológicos (Avitia y Uriarte, 2017). Se define como las habilidades que permiten al profesorado el desarrollo de la innovación en las acciones educativas (De Pablos, Colás, Conde y Reyes, 2017; Rodríguez, Trujillo y Sánchez, 2019), mediante el uso pedagógico de las TIC (Castañeda, Esteve y Adell, 2018).

La competencia digital está formada por cinco áreas: información y alfabetización informacional, comunicación y colaboración, creación de contenidos digitales, seguridad y resolución de problemas. Estas áreas se encuentran recogidas en el documento creado por el Instituto Nacional de Tecnologías Educativas y Formación del profesorado, bajo la denominación de marco común de competencia digital docente (INTEF, 2017).

Las recomendaciones dadas por el Parlamento Europeo sobre la formación y actualización del profesorado en conocimientos y habilidades tecnopedagógicos son tomadas en cuenta en el sistema educativo español (Sánchez, Ramos y Sánchez, 2014), orientadas al desarrollo de la competencia digital en el profesorado y estudiantes, con el propósito de lograr una educación que dé respuesta a las exigencias del nuevo milenio (Carrera y Coiduras, 2012; Gutiérrez 2014; Prendes y Gutiérrez, 2013; Medina, 2014; Rodríguez, Raso y Ruiz, 2019).

En base a investigaciones recientes sobre los planes formativos del profesorado, se deben tener presente la edad, el género, la experiencia docente, la posición laboral dentro de la institución, los recursos tecnológicos disponibles y las características sociales del centro educativo - entre los más destacados - debido a su influencia en el desarrollo de la competencia digital (Arrosagaray, González, Pino y Rodríguez, 2019; García y Cantón, 2019).
Actualmente, la formación de los docentes en competencia digital no es adecuada (López-Belmonte, Pozo-Sánchez, Vázquez-Cano y López-Meneses, 2020), dado que presentan destrezas dispares entre sí y entre las diversas áreas que componen dicha competencia (Afanador, 2017; Falcó, 2017; Fernández, Fernández y Rodríguez, 2018; Fernández y Rodríguez, 2017; Fuentes, López y Pozo, 2019). Todo ello propiciado por un déficit formativo y una actitud pasiva hacia la renovación de conocimientos (Prendes, Gutiérrez y Martínez, 2018), originando un plus de complejidad hacia la inclusión y desarrollo de las TIC en los espacios de aprendizaje (Cela, Esteve-González, Esteve-Mon, González y Gisbert, 2017).

Entre las diversas acciones pedagógicas que pueden desarrollar los propios docentes con el uso de las TIC se encuentra el aprendizaje electrónico, conocido comúnmente como e-learning, el cual se está extendiendo rápidamente en todos los ámbitos educativos (Cigdem y Topcu, 2015) y que se puede definir como el proceso formativo desarrollado de forma puramente virtual a través del uso de diversos recursos TIC (Moreno-Guerrero, Aznar-Díaz, Cáceres-Reche y Alonso-García, 2020), favoreciendo una comunicación síncrona o asíncrona y adecuando el espacio y tiempo a las necesidades de los agentes que intervienen, es decir, tanto docentes como discentes (Campos, Moreno-Guerrero y Soler, 2018; Venieris, Cohen, Vlismas, Naoum y Karatzimas, 2017).

El auge de estos métodos de enseñanza ha cobrado especial relevancia en los últimos años (Moreno-Guerrero, 2019), especialmente en la Formación Profesional (Dummert, Leber y Schwengler, 2019), la cual hace uso del e-learning para el desarrollo de ciclos formativos ofertados en la modalidad a distancia (Constantino y De Oliveira, 2017). Esta etapa educativa está siendo objeto de continuos cambios e investigaciones (López-Belmonte, Moreno-Guerrero, Pozo-Sánchez y López-Núñez, 2020; Wheelahan, 2015), debido a su capacidad para el desarrollo y transformación de la sociedad, así como de facilitar el camino hacia la equidad e inclusión de las personas (Sarmiento, Silva y Van Gameren, 2019).

Diversos estudios muestran que la combinación entre el enfoque e-learning y la Formación Profesional favorece el rendimiento (Brzezinska, 2018), la comunicación entre los diferentes colectivos (Callan, Johnston, Clayton y Poulsen, 2016; García y Cabero, 2016), el procesamiento y estructura de la información significativa (Kucheruk, Karaman, Karaman y Vinnikova, 2019) mediante el despliegue de acciones formativas no memorísticas-transmitivas (Cabreiro, Fernández y Arribi, 2017), basadas en la combinación de contenidos teóricos de alta calidad y actividades prácticas (Ugolini, Massetti, Sanesi y Pearlmutter, 2015; Widaningsih, Barliana, Aryanti y Malihah, 2018). No obstante, expertos como Dimache et al. (2015) establecen que las habilidades de carácter tecnopedagógico tanto de los docentes como de los discentes establecen un punto de inflexión en este enfoque innovador, marcando su éxito o fracaso en la Formación Profesional.

\subsection{Justificación y objetivos del estudio}

Debido a la situación sanitaria mundial generada por la pandemia COVID-19 que ha generado una gran incidencia en el sistema educativo (Almanthari, Maulina y Bruce, 2020) y a la proyección de numerosos recursos, aplicaciones y plataformas digitales en el campo de la educación así como al desarrollo de diferentes metodologías innovadoras de enseñanza y aprendizaje que garantizan la ubicuidad de la acción formativa, como es el caso del e-learning, surge esta investigación para seguir indagando y - por consiguiente - ampliar el campo de conocimiento en el seno de la Formación Profesional. 
El objetivo principal que se plantea es conocer si el profesorado de Formación Profesional dispone de competencias digitales para el despliegue de un enfoque de instrucción basado en el e-learning. Este objetivo general conduce a la formulación de los siguientes objetivos específicos, con el propósito de guiar la investigación:

- Concretar el nivel del profesorado en el área alusiva a la información y alfabetización informacional.

- Averiguar el nivel del profesorado en el área concerniente a la comunicación y colaboración.

- Conocer el nivel del profesorado en el área relativa a la creación de contenidos digitales.

- Determinar el nivel del profesorado en el área conexa a la seguridad en el plano digital.

- Identificar el nivel del profesorado en el área relacionada con la resolución de problemas.

- Definir el grado de utilización del e-learning en el profesorado de Formación Profesional.

- Conocer los motivos por los que el profesorado no emplea el $e$-learning.

- Predecir la incidencia del nivel de competencia digital de los docentes en el uso del e-learning

A raíz de estos objetivos surgen las siguientes preguntas de investigación:

- $\mathrm{PI}_{1}$ : ¿Cuál/es de las cinco áreas de la competencia digital presentan mayor influencia en el uso del e-learning?

- $\mathrm{PI}_{2}$ : ¿Está capacitado el profesorado de Formación Profesional para llevar a cabo un proceso formativo mediante e-learning?

- $\mathrm{PI}_{3}$ : ¿Determina el nivel de competencia digital docente el uso del e-learning por parte del profesorado?

\section{Método}

\subsection{Diseño de investigación y variables}

Este estudio se ha llevado a cabo a través de un diseño no experimental de tipo descriptivo, correlacional y predictivo, fundamentado en un método cuantitativo para el tratamiento de los datos, siguiendo los preceptos de Hernández, Fernández y Baptista (2014).

Las variables que se han analizado en esta investigación se encuentran recopiladas en este apartado, junto con su codificación con la intención de mejorar la presentación e interpretación de los resultados. Las variables definidas son las siguientes: Nivel de destrezas en información y alfabetización digital (ÁREA1); Nivel de destrezas en comunicación y colaboración (ÁREA2); Nivel de destrezas en creación de contenidos digitales (ÁREA3); Nivel de destrezas en seguridad (ÁREA4); Nivel de destrezas en resolución de problemas (ÁREA5); Frecuencia de utilización del e-learning (FREL); Déficit formativo en competencia digital (DFCD); Falta de confianza en el e-learning (FCEL); Ausencia de recursos tecnológicos (ART); Enfoque no compatible con el alumnado (ENCA); Negativa hacia el uso de las TIC en el proceso de instrucción (NTIC).

\subsection{Participantes}

Los sujetos de estudio han constituido una muestra de 627 docentes españoles que se encuentran desarrollando su profesión en la etapa de Formación Profesional. De estos participantes, el $73,52 \%$ son hombres y el $26,48 \%$ son mujeres, con una media de edad de 43 años (DT=11,82).

Estos profesionales de la educación han sido seleccionados a través de un muestreo probabilístico simple, partiendo de la población total española ( $\mathrm{N}=4257)$ de centros educativos de Formación Profesional, en sus distintas tipologías (públicos=2595; privados=811; concertados=851). Estos datos han sido extraídos de la base de datos que ofrece de manera abierta y online el Ministerio de Educación y Formación Profesional (https://bit.ly/2TzPFiY). Se escogieron 2650 docentes, abarcando un total de 530 centros educativos con ciclos formativos de Formación Profesional, de los que finalmente solo el $23,66 \%$ de los sujetos $(n=627)$ participaron en el estudio.

\subsection{Instrumento}

La recogida de datos se ha efectuado a través de un cuestionario de naturaleza ad hoc. Para su confección se ha partido de otros cuestionarios validados sobre competencia digital hallados en la literatura científica (Agreda, Hinojo y Sola, 2016; Tourón, Martín, Navarro, Pradas e Íñigo, 2018). La herramienta confeccionada integra un total de 66 ítems que han sido catalogados en diferentes dimensiones: a) Socioeducativa (8 ítems); b) Información y alfabetización informacional (9 ítems); c) Comunicación y colaboración (8 ítems); d) Creación de contenidos digitales (20 ítems); e) Seguridad (14 ítems); f) Resolución de problemas (7 ítems).

Las distintas cuestiones están configuradas principalmente con una escala Likert de 5 puntos (1-Muy bajo; 2-Bajo; 3-Medio; 4-Alto; 5-Muy alto) y otras cuestiones - por su naturaleza - siguen un patrón de selección cerrada, dicotómica y de opinión abierta.

Para validar el cuestionario de manera cualitativa se ha empleado un método Delphi (Cabero e Infante, 2014), con el que optimizar el instrumento a través de los juicios emitidos por 6 especialistas en el ámbito de las TIC, quienes ofrecieron una retroalimentación objetiva y anónima que sirvió para mejorar la estructura y formulación de los ítems del cuestionario. Asimismo, se realizaron diversos procedimientos estadísticos para verificar la pertinencia y concordancia de las observaciones de los expertos, mediante las pruebas Kappa de Fleiss y W de Kendall que resultaron adecuadas $(\mathrm{K}=0,81 ; \mathrm{W}=0,84)$.

La validación cuantitativa se materializó por medio de un análisis factorial exploratorio, siguiendo el método de componentes principales con una rotación varimax. Se obtuvo dependencia entre las variables con el test de esfericidad (Bartlett=2765,37; $p$ $<0,001)$. Por otro lado, el test de Kaiser-Meyer-Olkin reveló una puntuación pertinente $(\mathrm{KMO}=0,92)$ sobre la adecuación muestral.

Seguidamente, se calculó la fiabilidad media del cuestionario con diferentes estadísticos como el alfa de Cronbach $(\alpha=0,85)$, la fiabilidad compuesta $(\mathrm{FC}=0,84)$ y la varianza media extractada (VME=0,77), obteniéndose valores que afirman adecuados índices de fiabilidad del instrumento en cuestión (Bisquerra, 2004).

\subsection{Procedimiento}

El despliegue investigador tuvo su origen en el 2019, teniendo como primera fase el diseño y validación del cuestionario para recopilar los datos. Seguidamente se produjo la selección de la muestra tomando como referencia la información obtenida de la base de datos del Ministerio de Educación y Formación Profesional. El envío del cuestionario se produjo de forma telemática y en el mismo se obtuvo el consentimiento informado de los participantes. La fase de cumplimentación del cuestionario se prolongó durante cinco meses, tiempo en el que los investigadores se encontraron activos para atender las inquietudes de los distintos participantes. A continuación, se prepararon los datos para ser importados al programa estadístico y realizar un análisis en profundidad, abarcando las distintas variables presentadas. 


\subsection{Análisis de datos}

Para desarrollar este estudio se han utilizado estadísticos básicos como la media (M) y la desviación típica (DT), así como pruebas específicas para determinar la tendencia de la distribución como el coeficiente de asimetría de Pearson (CAP) y el de apuntamiento de Fisher (CAF). La asociación de las variables se ha realizado mediante el test Chi-cuadrado de Pearson $\left(\chi^{2}\right)$, junto con la V de Cramer (V) y el coeficiente de contingencia (Cont) para concretar el índice de fuerza asociativa. Además, se ha realizado un modelo de regresión lineal múltiple con el propósito de predecir el efecto de cada una de las áreas de la competencia digital docente sobre el uso del e-learning.

Asimismo, se ha efectuado un análisis clúster bietápico, asumiendo un $5 \%$ de error, para clasificar los participantes en grupos similares derivados de un conjunto supuestamente heterogéneo (Rubio y Vilà, 2017). Los criterios de agrupamiento fueron: a) El grado competencial en cada área de la CDD, conformando cinco conglomerados (1-Muy Bajo; 2-Bajo; 3-Medio; 4-Alto; 5-Muy alto); b) El grado de utilización del e-learning, originando cuatro conglomerados (1-Nunca; 2-Esporádicamente; 3-Frecuentemente; 4-Siempre).

El programa utilizado para analizar los datos y efectuar las distintas pruebas ha sido el Statistical Package for the Social Sciences (SPSS) en su versión 24, determinando diferencias estadísticamente significativas los valores de $p<0,05$.

\section{Resultados}

En este apartado se presentan los resultados obtenidos tras el estudio de las diferentes variables establecidas con el fin de alcanzar los objetivos formulados. La información se plasma en figuras y tablas para facilitar la interpretación de los hallazgos. En primer lugar, en la figura 1 se muestra la frecuencia de utilización del enfoque formativo e-learning por parte de los docentes analizados. En ella se aprecia una distribución escalonada, en la que destaca que más de la mitad de estos profesionales $(57,74 \%)$ no lo utiliza como metodología de enseñanza y aprendizaje en su praxis diaria. Con menor índice de frecuencias le sigue el resto de grupos de docentes que lo emplean de manera esporádica $(21,37 \%)$ y de forma frecuente $(11,64 \%)$, siendo muy pocos los profesionales que utilizan este enfoque cotidianamente $(9,25 \%)$.

Los motivos manifestados por los docentes para no utilizar este enfoque innovador quedan recogidos en la figura 2. Tal y como se visualiza, predomina la razón alusiva a las carencias formativas de los docentes en el ámbito digital (40,61\%), acompañada de la falta de confianza en dicho método de instrucción $(30,11 \%)$. En menor grado, se encuentran otros factores como el rechazo hacia las TIC $(15,47 \%)$, la ausencia de recursos, medios

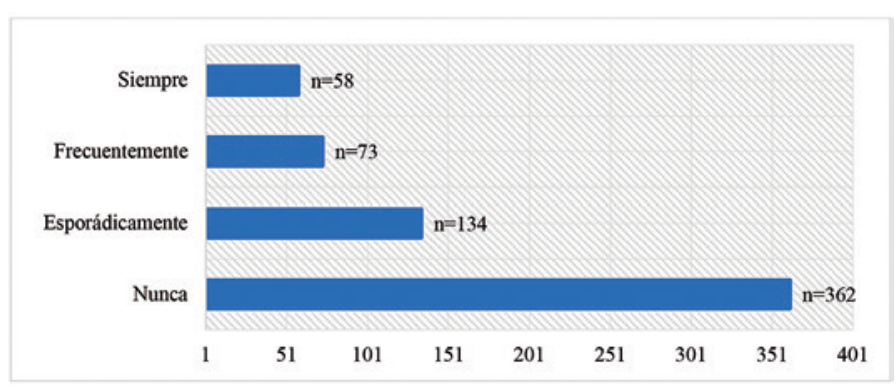

Figura 1. Utilización del e-learning por parte del profesorado y equipamientos tecnológicos $(10,77 \%)$ y - por último - por su escasez de frecuencias se encuentra la causa relativa a la incompatibilidad con el alumnado (3,04\%).

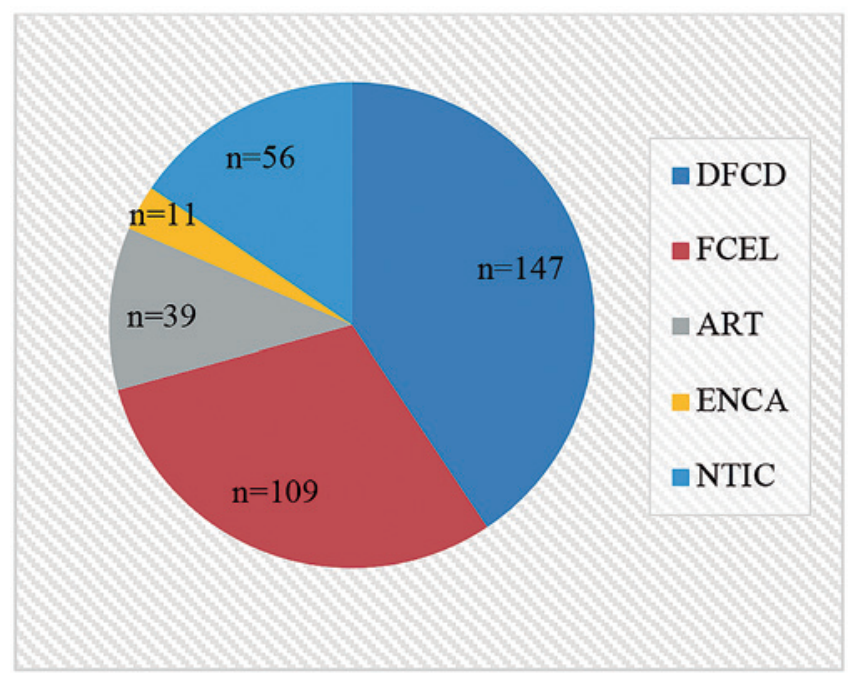

Figura 2. Motivos por los que el profesorado no utiliza el e-learning

El análisis de cada una de las áreas que configuran la competencia digital docente queda recogido en la tabla 1. Las puntuaciones alcanzadas reflejan un déficit formativo del profesorado en la vertiente tecnopedagógica. Los resultados conforman una distribución asimétrica con tendencia a la izquierda, debido a los valores concentrados en los estadios más carentes de la escala de medición empleada. Las cifras más elevadas se han hallado en las áreas relativas a la información y alfabetización informacional y en la comunicación y colaboración de los docentes. Sin embargo, su puntaje se aglutina en el segundo rango de la escala, definido como un nivel de destreza bajo. Las demás áreas revelan puntuaciones muy deficientes, halladas - principalmente - en el primer rango de valoración. La realización de la media totalizada verifica un bajo nivel de competencia digital de los participantes, ocasionando un relevante déficit de destrezas concernientes a la intersección entre lo tecnológico y pedagógico.

Con respecto al estudio asociativo entre la frecuencia de utilización del e-learning teniendo en cuenta los distintos niveles de destrezas en cada una de las áreas que conforman la competencia digital de profesorado, la tabla 2 muestra la aparición de diferencias estadísticamente significativas en dos áreas competenciales, concretamente en la alusiva a la información y alfabetización informacional y en la comunicación y colaboración. En la primera de ellas, el tamaño del efecto $(E S)$ es elevado $(V>0,6)$ lo que revela una intensa fuerza de asociación entre las variables analizadas. En cambio, la intensidad asociativa en la comunicación y colaboración es moderada $(0,2<\mathrm{ES}<0,6)$. Todo esto hace indicar que el profesorado con mejores niveles competenciales en las áreas citadas desarrolla con mayor frecuencia una praxis docente mediante e-learning. El resto de áreas no han mostrado indicios de significancia estadística sobre la frecuencia de uso de este enfoque metodológico.

Mediante el modelo de regresión lineal múltiple se trató de identificar el efecto de la competencia digital de los docentes sobre el uso del e-learning. En este caso, se determinó por aplicar el modelo de pasos sucesivos. De dicho análisis se obtuvieron un total de tres modelos, siendo el modelo 3 el que ofrece valores 
Tabla 1.

Descriptivo de las puntuaciones obtenidas en las áreas de la CDD

\begin{tabular}{|c|c|c|c|c|c|c|c|c|c|}
\hline & \multicolumn{5}{|c|}{ Escala Likert $n(\%)$} & \multicolumn{4}{|c|}{ Parámetros } \\
\hline & Muy bajo & Bajo & Medio & Alto & Muy alto & $\mathbf{M}$ & DT & CAP & CAF \\
\hline ÁREA1 & $45(7,17)$ & $103(16,42)$ & $214(34,13)$ & $177(28,22)$ & $88(14,03)$ & 2,25 & 1,109 & 1,131 & $-0,584$ \\
\hline ÁREA2 & $27(4,3)$ & $87(13,87)$ & $223(35,56)$ & $192(30,62)$ & $98(15,62)$ & 2,39 & 1,042 & 1,336 & $-0,438$ \\
\hline ÁREA3 & $99(15,78)$ & $175(27,91)$ & $164(26,15)$ & $103(16,42)$ & $86(13,71)$ & 1,84 & 1,264 & 0,667 & $-0,956$ \\
\hline ÁREA4 & $87(13,87)$ & $159(25,35)$ & $173(27,59)$ & $123(19,61)$ & $85(13,55)$ & 1,93 & 1,242 & 0,753 & $-0,96$ \\
\hline ÁREA5 & $92(14,67)$ & $153(24,4)$ & $155(24,72)$ & $136(21,69)$ & $91(14,51)$ & 1,96 & 1,275 & 0,760 & $-1,061$ \\
\hline Total & $350(11,09)$ & $667(21,14)$ & $956(30,31)$ & $731(23,17)$ & $448(14,21)$ & 1,9 & 1,187 & 0,093 & $-0,801$ \\
\hline
\end{tabular}

Tabla 2.

Asociación entre FREL con cada una de las áreas de la CDD

\begin{tabular}{|c|c|c|c|c|c|c|c|c|}
\hline \multirow[b]{2}{*}{ Likert } & \multicolumn{4}{|c|}{ FREL $n(\%)$} & \multicolumn{4}{|c|}{ Parámetros } \\
\hline & Nunca & Esporádico & Frecuente & Siempre & $\chi^{2}(g l)$ & $p$-valor & Cont & V \\
\hline ÁREA1 & & & & & $120,3(12)$ & $<0,001$ & 0,401 & 0,759 \\
\hline Muy bajo & $30(4,78)$ & $10(1,59)$ & $4(0,63)$ & $1(0,15)$ & & & & \\
\hline Bajo & $67(10,68)$ & $29(4,62)$ & $4(0,63)$ & $3(0,47)$ & & & & \\
\hline Medio & $153(24,4)$ & $42(6,69)$ & $17(2,71)$ & $2(0,31)$ & & & & \\
\hline Alto & $85(13,55)$ & $39(6,22)$ & $29(4,62)$ & $24(3,82)$ & & & & \\
\hline Muy alto & $27(4,3)$ & $14(2,23)$ & $19(3,03)$ & $28(4,46)$ & & & & \\
\hline ÁREA2 & & & & & 31,15 (12) & 0,002 & 0,218 & 0,386 \\
\hline Muy bajo & $14(2,23)$ & $10(1,59)$ & $2(0,31)$ & $1(0,15)$ & & & & \\
\hline Bajo & $44(7,01)$ & $27(4,3)$ & $11(1,75)$ & $5(0,79)$ & & & & \\
\hline Medio & $122(19,45)$ & $51(8,13)$ & $30(4,78)$ & $20(3,18)$ & & & & \\
\hline Alto & $129(20,57)$ & $25(3,98)$ & $13(2,07)$ & $25(3,98)$ & & & & \\
\hline Muy alto & $53(8,45)$ & $21(3,34)$ & $17(2,71)$ & $7(1,11)$ & & & & \\
\hline ÁREA3 & & & & & $18,91(12)$ & 0,091 & 0,171 & 0,301 \\
\hline Muy bajo & $61(9,72)$ & $16(2,55)$ & $11(1,75)$ & $11(1,75)$ & & & & \\
\hline Bajo & $99(15,78)$ & $43(6,85)$ & $22(3,5)$ & $11(1,75)$ & & & & \\
\hline Medio & $99(15,78)$ & $32(5,1)$ & $12(1,91)$ & $21(3,34)$ & & & & \\
\hline Alto & $54(8,61)$ & $23(3,66)$ & $13(2,07)$ & $13(2,07)$ & & & & \\
\hline Muy alto & $49(7,81)$ & $20(3,18)$ & $15(2,39)$ & $2(0,31)$ & & & & \\
\hline ÁREA4 & & & & & $3,73(12)$ & 0,988 & 0,077 & 0,134 \\
\hline Muy bajo & $54(8,61)$ & $17(2,71)$ & $8(1,27)$ & $8(1,25)$ & & & & \\
\hline Bajo & $97(15,47)$ & $32(5,1)$ & $17(2,71)$ & $13(2,07)$ & & & & \\
\hline Medio & $96(15,31)$ & $38(6,06)$ & $24(3,82)$ & $15(2,39)$ & & & & \\
\hline Alto & $66(10,52)$ & $29(4,62)$ & $15(2,39)$ & $13(2,07)$ & & & & \\
\hline Muy alto & $49(7,81)$ & $18(2,87)$ & $9(1,43)$ & $9(1,43)$ & & & & \\
\hline ÁREA5 & & & & & 7,95 (12) & 0,789 & 0,112 & 0,195 \\
\hline Muy bajo & $61(9,72)$ & $16(2,55)$ & $9(1,43)$ & $6(0,95)$ & & & & \\
\hline Bajo & $93(14,83)$ & $32(5,1)$ & $15(2,39)$ & $13(2,07)$ & & & & \\
\hline Medio & $86(13,71)$ & $35(5,58)$ & $21(3,34)$ & $13(2,07)$ & & & & \\
\hline Alto & $70(11,16)$ & $34(5,42)$ & $17(2,71)$ & $15(2,39)$ & & & & \\
\hline Muy alto & $52(8,29)$ & $17(2,71)$ & $11(1,75)$ & $11(1,75)$ & & & & \\
\hline
\end{tabular}


Tabla 3.

Modelo de regresión lineal múltiple por pasos sucesivos

\begin{tabular}{|c|c|c|c|c|c|c|c|}
\hline Modelos & $\mathbb{R}$ & $\mathbb{R} 2$ & $\mathbb{R} 2$ C & ETE & CR2 & SCF \\
\hline 1 & 0,851 & 0,724 & 0,723 & 0,523 & 0,724 & 1636,37 & 0,000 \\
\hline 2 & 0,857 & 0,735 & 0,734 & 0,513 & 0,012 & 27,173 & 0,000 \\
\hline 3 & 0,860 & 0,740 & 0,738 & 0,509 & 0,005 & 10,848 \\
\hline
\end{tabular}

Tabla 4.

Coeficientes de la regresión lineal múltiple del modelo 3

\begin{tabular}{|c|c|c|c|c|c|}
\cline { 2 - 5 } \multicolumn{1}{c|}{} & B & Error Típico & Beta & Sig. & 9,841 \\
\hline 3(Constante) & 0,780 & 0,079 & & 0,000 \\
\hline ÁREA1 & 0,375 & 0,066 & 0,418 & 0,691 \\
\hline ÁREA5 & 0,177 & 0,048 & 0,227 & 3,678 \\
\hline ÁREA2 & 0,221 & 0,067 & 0,232 & 3,000 \\
\hline
\end{tabular}

estadísticos más significativos. Este modelo explicar un $86 \%$ de la varianza. Es decir, las dimensiones que componen el modelo 3 explican en dicho porcentaje el efecto que tienen la competencia digital de los docentes para un desarrollo y aplicación del método e-learning. Si se atiende a la R2 corregida, se puede indicar que las dimensiones del modelo 3 tienen una capacidad predictiva del $73,8 \%$ (tabla 3 ).

El modelo 3 está compuesto por 3 dimensiones: el nivel de destrezas en información y alfabetización informacional (ÁREA1), el nivel de destrezas en resolución de problemas (ÁREA5), y el nivel de destrezas en comunicación y colaboración (ÁREA2). Estas dimensiones muestran valores de significancia inferiores a 0,05. Mediante el valor $\mathrm{t}$ se muestra que estas dimensiones favorecen la explicación de la varianza, teniendo capacidad predictiva. Además, todos los valores obtenidos son positivos (tabla 4).

\section{Discusión y conclusiones}

La renovación pedagógica requiere de un docente que tenga adquirido un adecuado nivel de competencia digital y sea capaz de llevar a cabo un desarrollo profesional de la mano de recursos, aplicaciones y medios tecnológicos, tal y como establecieron De Pablos et al. (2017), Elche y Yubero (2019), López et al. (2019) y Rodríguez et al. (2019), aspectos que el profesorado de esta investigación no logra alcanzar.

Esto exige una formación constante y continua de los docentes en la aplicación y desarrollo de las TIC, como ya marcaron Castañeda et al. (2018) y Larionova et al. (2018), algo que se está produciendo mediante programas formativos ofertados por diversos organismos para la mejora y adquisición de la competencia digital, según establecieron Carrera y Coiduras (2012), Gutiérrez (2014), Prendes y Gutiérrez (2013), Medina (2014) y Rodríguez et al. (2019). A pesar de ello, estos no están dando los resultados esperados, debido a las carencias que muestran los docentes en las cinco áreas analizadas de la competencia digital (Afanador, 2017; Falcó, 2017; Fernández et al., 2018; Fernández y Rodríguez, 2017; Fuentes et al., 2019). Estos hallazgos reportados de la literatura especializada se encuentran en analogía con los resultados obtenidos en la presente investigación.

En este estudio, centrado en el colectivo docente de Formación Profesional, se ha obtenido que las destrezas competenciales de tales profesionales se sitúan en niveles muy deficientes, excepto en las áreas alusivas a la información y alfabetización de la información y a la comunicación y colaboración, donde los niveles son ligeramente superiores al resto, aunque siguen siendo insuficientes para un despliegue formativo innovador.

En la Formación Profesional se requiere de la aplicación de métodos activos de enseñanza - dado el carácter práctico de los ciclos formativos - siendo primordial la adquisición de destrezas tecnopedagógicas para fomentar la entrada y expansión de la tecnología educativa en los espacios de aprendizaje, como postularon Cela et al. (2017).

El uso de las TIC ha aumentado en los últimos tiempos (Dummert et al., 2019; Moreno-Guerrero, 2019), estando los discentes familiarizados con su uso ya que han crecido y convivido desde su infancia con la tecnología (Rodríguez et al., 2017). Dentro de la propia aplicación de los métodos activos de enseñanza, se encuentra el e-learning, usado principalmente en esta etapa educativa para el desarrollo de aquellos ciclos formativos que se imparten desde una modalidad a distancia. En este estudio los hallazgos permiten determinar que menos de la mitad del profesorado encuestado (42,26\%) usa el e-learning en su labor docente, justificando el resto su nula utilización en sus carencias formativas en el ámbito digital, tal y como ya concluyeron Fuentes et al. (2019) y su falta de confianza en el uso de este método de enseñanza, estando este motivo en contraposición a lo establecido en investigaciones anteriores donde se resalta una mejora del rendimiento (Brzezinska, 2018), de la comunicación (Callan et al. 2016; García y Cabero, 2016) y del procesamiento la información (Kucheruk et al., 2019).

Aunque la literatura refleja ventajas relacionadas con el uso y aplicación de este método innovador, solamente un $9.25 \%$ lo emplea de forma diaria $-\mathrm{y}$ tal y como se observa en los resultados de este estudio - por aquellos docentes con mejores niveles competenciales en el ámbito digital. Este hecho se encuentra en consonancia con las afirmaciones de Dimache et al. (2015), los cuales establecieron que las habilidades de carácter tecnopedagógico en los docentes establecen un punto de inflexión en la aplicación de este método.

Finalmente, se puede establecer que las áreas competenciales relacionadas con la información y alfabetización informacional, la resolución de problemas y las destrezas en comunicación y colaboración pueden determinar el uso o no del método e-learning en 
el profesorado de Formación Profesional. En este caso, si el profesorado no presenta niveles de competencia digital adecuados en estas áreas, no suele hacer uso del método formativo indicado.

En base a los resultados alcanzados tras el análisis estadístico, se dan respuesta a los interrogantes planteados en el estudio:

- $\mathrm{PI}_{1}$ : Las áreas de la competencia digital más influyentes en el uso del e-learning en el profesorado de Formación Profesional han sido las relativas a la información y alfabetización informacional y a la comunicación y colaboración, alcanzándose en ambas una significancia a nivel estadístico.

- $\mathrm{PI}_{2}$ : El profesorado de Formación Profesional no revela niveles competenciales óptimos que le permita - por un ladodesplegar una praxis educativa eficaz mediante $e$-learning y - por otro- aprovechar todas las potencialidades tanto del uso de las TIC como de tal método innovador en concreto.

- $\mathrm{PI}_{3}$ : Los niveles de competencia digital del profesorado marcan su uso o no del método e-learning. En este caso, se requiere de niveles competenciales en la información y alfabetización informacional, en la resolución de problemas y en las destrezas de comunicación y colaboración. Con niveles competenciales adecuados en estas tres áreas, el profesorado de Formación Profesional puede hacer uso del método e-learning.

La prospectiva que deriva de este estudio se focaliza en la promoción de programas formativos que potencien la adquisición y mejora de cada una de las distintas áreas de la competencial digital docente con el fin de lograr una mayor proyección del e-learning en la etapa de Formación Profesional, sobre todo en la actualidad, debido a la incidencia que la pandemia del COVID-19 ha tenido en el sistema educativo. Igualmente, la acción formativa debe lograr satisfacer la demanda y exigencias del profesorado, adecuando sus contenidos a la realidad actual de los espacios de aprendizaje.

La principal limitación de esta investigación se concentra en la participación del profesorado, siendo necesaria la constancia y perseverancia de los investigadores para recopilar los datos mostrados en este trabajo. Como futura línea de investigación -con la finalidad de seguir indagando en este contexto específico - se pretende estudiar la incidencia de variables socioeducativas como el sexo y los años de experiencia en la utilización del e-learning.

\section{Conflicto de intereses}

Los autores declaran no tener ningún conflicto de intereses.

\section{Agradecimientos}

A todos los miembros del Grupo de Investigación AREA (HUM-672) de la Universidad de Granada.

\section{Referencias bibliográficas}

Afanador, H. A. (2017). Estado actual de las competencias TIC de docentes. Puente, 9(2), 23-32.

Agreda, M., Hinojo, M. A., y Sola, J. M. (2016). Diseño y validación de un instrumento para evaluar la competencia digital de los docentes en la Educación Superior española. Pixel-Bit: Revista de Medios y Educación, 49, 39-56. https://www.doi.org/10.12795/ pixelbit.2016.i49.03

Almanthari, A., Maulina, S., y Bruce, S. (2020). Secondary School Mathematics Teachers' Views on E-learning Implementation Barriers during the COVID-19 Pandemic: The Case of Indonesia. Eurasia Journal of Mathematics, Science and Technology Education, 16(7), 1-9. https://www.doi.org/10.29333/ejmste/8240
Area, M. (2015). La alfabetización digital y la formación de la ciudadanía del siglo XXI. Revista Integra Educativa, 7(3), 21-33.

Area, M., Hernández, V., y Sosa, J. J. (2016). Modelos de integración didáctica de las TIC en el aula. Comunicar: Revista científica iberoamericana de comunicación y educación, 24(47), 79-87. https:// www.doi.org/10.3916/C47-2016-08

Arrosagaray, M., González, M., Pino, M., y Rodríguez, B. (2019). A comparative study of Spanish adult students' attitudes to ICT in classroom blended and distance language-learning modes. Computers $\mathcal{E}$ Education, 134, 31-40. https://www.doi. org/10.1016/j.compedu.2019.01.016

Avitia, P., y Uriarte, I. (2017). Evaluación de la habilidad digital de los estudiantes universitarios: estado de ingreso y potencial educativo. EDUTEC. Revista Electrónica de Tecnología Educativa, (61), 1-13.

Bisquerra, R. (2004). Metodología de la investigación educativa. Madrid: La Muralla.

Brzezinska, M. (2018). An intuitive app generator and accompanying e-learning modules as examples of successful assistive technology. E-mentor, (2), 19-26. https://www.doi.org/10.15219/ em74.1348

Cabero, J., e Infante, A. (2014). Empleo del método Delphi y su empleo en la investigación en comunicación y educación. Edutec, 48, 1-16. https://www.doi.org/10.21556/edutec.2014.48.187

Callan, V.J., Johnston, M.A., Clayton, B., y Poulsen, A.L. (2016). E-assessment: challenges to the legitimacy of VET practitioners and auditors. Journal of vocational education and training, 68(4), 416-435. https://www.doi.org/10.1080/13636820.2016.1 231214

Campo, M.N., Moreno-Guerrero, A.J., y Soler, R. (2018). Use of Google Drive and Whatsapp for the follow-up and development of the final Master's Project through m-learning. The Turkish Online Journal of Educational Technology, 2, 858-864.

Carrera, F. X., y Coiduras, J. L. (2012). Identificación de la competencia digital del profesor universitario: un estudio exploratorio en el ámbito de las ciencias sociales. REDU - Revista de Docencia Universitaria. 10(2), 273-298. https://www.doi. org/10.4995/redu.2012.6108

Castañeda, L., Esteve, F., y Adell, J. (2018). ¿Por qué es necesario repensar la competencia docente para el mundo digital? RED. Revista de Educación a Distancia, 56, 1-20. https://www. doi.org/10.6018/red/56/6

Cebreiro, B., Fernández, C., y Arribi, J. (2017). Distance vocational training: running in the wrong direction. Pixel-Bit. Revista de Medios y Educación, (50), 65-76. https://www.doi.org/10.12795/ pixelbit.2017.i50.04

Cela, J. M., Esteve-González, V., Esteve-Mon, F., González, J., y Gisbert, M. (2017). El docente en la sociedad digital: Una propuesta basada en la pedagogía transformativa y en la tecnología avanzada. Profesorado. Revista de Currículum y Formación del Profesorado, 21(1), 403-422.

Cigdem, H., y Topcu, A. (2015). Predictors of instructors' behavioral intention to use learning management system: A Turkish vocational college example. Computers in human behaviour, 52, 22-28. https://www.doi.org/10.1016/j.chb.2015.05.049

Constantino, P., y De Oliveria, M.G., (2017). E-learning in vocational education: a portrait of offer and public policies for state of Sao Paulo, Brazil. Revista Ibero-americana de estudios em Educaçao, 12, 1234-1242. https://www.doi.org/10.21723/riaee. v12.n.esp.2.10292

De Pablos, J., Colás, P., Conde, J., y Reyes, S. R. (2017). La competencia digital de los estudiantes de educación no universitaria: variables predictivas. Bordón. Revista de pedagogía, 69(1), 169185. https://www.doi.org/10.13042/Bordon.2016.48594 
Dimache, A., Roche, T., Kopeinik, S., Winter, L.C., Nussbaumer, A., y Albert, D. (2015). Suitability of Adaptive Self-Regulated e-Learning to Vocational Training: A Pilot Study in Heat Pump System Installation. International Journal of Online Pedagogy and Course Design, 5(3), 31-46. https://www.doi. org/10.4018/ijopcd.2015070103

Dummert, S., Leber, U., y Schwengler, B. (2019). Unfilled Training Positions in Germany - Regional and Establishment-Specific Determinants. Jahrbücher für Nationalökonomie und Statistik, 239(4), 661-701. https://www.doi.org/10.1515/jbnst-2018-0014

Elche, M., y Yubero, S. (2019). The influence of reading habits on the use of internet: a study with university students. Investigación bibliotecológica, 33(79), 51-66. https://www.doi. org/10.22201/iibi.24488321xe.2019.79.57985

Falcó, J. M. (2017). Evaluación de la competencia digital docente en la Comunidad Autónoma de Aragón. Revista electrónica de investigación educativa, 19(4), 73-83.

Fernández, F. J., Fernández, M. J., y Rodríguez, J. M. (2018). El proceso de integración y uso pedagógico de las TIC en los centros educativos madrileños. Educación XX1, 21(2), 395-416. https://www.doi.org/10.5944/educxx1.17907

Fernández, J. M., y Rodríguez, A. (2017). TIC y diversidad funcional: conocimiento del profesorado. EJIHPE. European Journal of Investigation in Health, Psychology and Education, 7(3), 157-175. https://www.doi.org/10.1989/ejihpe.v7i3.203

Fuentes, A., López, J., y Pozo, S. (2019). Analysis of the Digital Teaching Competence: Key Factor in the Performance of Active Pedagogies with Augmented Reality. REICE. Revista Iberoamericana sobre calidad, eficacia y cambio en educación, 17(2), 27-42. https://www.doi.org/10.15366/reice2019.17.2.002

García, C.J., y Cabero, J. (2016). The development and current situation of e-learning in Spanish Vocational Training. RIED. Revista Iberoamericana de Educación a Distancia, 19(2), 167-191. https://www.doi.org/10.5944/ried.19.2.15800

García, S., y Cantón, I. (2019). Teachers 3.0: Patterns of Use of Five Digital Tools. Digital Education Review, (35), 202-215. https:// www.doi.org/10.1344/der.2019.35.202-215

Gutiérrez, I. (2014). Perfil del profesor universitario español en torno a las competencias en TIC. Pixel-Bit. Revista de Medios y Educación, 44, 51-65. https://www.doi.org/10.12795/pixelbit.2014.i44.04

Hernández, R., Fernández, C., y Baptista, M. P. (2014). Metodología de la investigación. Madrid: McGraw Hill.

INTEF (2017). Marco de Competencia Digital. Madrid: Ministerio de Educación, Ciencia y Deportes.

Kucheruk, O.A., Karaman, S.A., Karaman, O.V., y Vinnikova, N.M. (2019). Using ICT tolos for forming profesional competences of future teachers of the Ukrainian language and literature. Information Technologies and Learning Tools, 71(3), 196-214. https://www.doi.org/10.33407/itlt.v71i3.2814

Larionova, V., Brown, K., Bystrova, T., y Sinitsyn, E. (2018). Russian perspectives of online learning technologies in higher education: An empirical study of a MOOC. Research in comparative and international education, 13(1), 70-91. https://www. doi.org/10.1177/1745499918763420

López, J., Pozo, S., Fuentes, A., y Romero, J. M. (2019). Analysis of electronic leadership and digital competence of teachers of educational cooperatives in Andalucia (Spain). REMIE. Multidisciplinary Journal of Educational Research, 9(2), 194-223. https:// www.doi.org/10.4471/remie.2019.4149

López-Belmonte, J., Moreno-Guerrero, A. J., Pozo-Sánchez, S., y López-Nuñez, J. A. (2020). Efecto de la competencia digital docente en el uso del blended learning en formación profesional. Investigación Bibliotecológica: archivonomía, bibliotecología e información, 34(83), 187-205. https://www.doi.org/10.22201/ iibi.24488321xe.2020.83.58147

López-Belmonte, J., Pozo-Sánchez, S., Vázquez-Cano, E., y López-Meneses, E. (2020). Análisis de la incidencia de la edad en la competencia digital del profesorado preuniversitario español. Revista Fuentes, 22(1), 86-100. https://www.doi. org/10.12795/revistafuentes.2020.v22.i1.07

Medina, J.A. (2014). Competencias en las nuevas tecnologías requeridas por los docentes. Saarbrücken, Alemania: Publicia.

Moreira, E., García, M., Conde, A., y González, A. (2019). Teachers' ICT-related self-efficacy, job resources, and positive emotions: Their structural relations with autonomous motivation and work engagement. Computers $\mathcal{E}$ Education, 134, 63-77. https://www.doi.org/10.1016/j.compedu.2019.02.007

Moreno-Guerrero, A.J. (2019). Estudio bibliométrico de la producción científica en Web os Science. Formación Profesional y blended learning. Pixel-Bit. Revista de medios y educación, (56), 149-168. https://www.doi.org/10.12795/pixelbit.2019.i56.08

Moreno-Guerrero, A.J., Aznar-Díaz, I., Cáceres-Reche, P., y Alonso-García, S. (2020). E-Learning in the Teaching of Mathematics: An Educational Experience in Adult High School. Mathematics, 8(5), 1-16. https://www.doi.org/10.3390/math8050840

Prendes, M. P., Gutiérrez, I. y Martínez, F. (2018). Competencia digital: una necesidad del profesorado universitario en el siglo XXI. RED: Revista de Educación a Distancia, (56), 1-22.

Prendes, M.P., y Gutiérrez, I. (2013): Competencias tecnológicas del profesorado en las universidades españolas. Revista de Educación, 361, 196-222. https://www.doi.org/10.4438/1988592X-RE-2011-361-140

Rodríguez, A.M., Raso, F., y Ruiz, J. (2019). Digital competence, higher education and teacher training: a metaanalysis study on the Web of Science. Pixel Bit. Revista de Medios y Educación, (54), 65-81. https://www.doi.org/10.12795/pixelbit.2019.i54.04

Rodríguez, A.M., Romero, J.M., y Agreda, M. (2019). Impact of ICT on the teaching of Physical Education: a bibliometric research study. ESHPA - Education, Sport, Health and Physical Activity, 3(1), 1-14. http://hdl.handle.net/10481/53211

Rodríguez, A.M., Trujillo, J.M., y Sánchez, J. (2019). Impacto de la productividad científica sobre competencia digital de los futuros docentes: aproximación bibliométrica en Scopus y Web of Science. Revista complutense de educación, 30(2), 623-646. https:// www.doi.org/10.5209/RCED.58862

Rubio, M. J., y Vilà, R. (2017). El análisis de conglomerados bietápico o en dos fases con SPSS. REIRE. Revista d'Innovació $i$ Recerca en Educació, 10(1), 118-126. https://www.doi.org/10.1344/ reire2017.10.11017

Sánchez, P., Ramos, F.J., y Sánchez, J. (2014). Formación continua y competencia digital docente: el caso de la comunidad de Madrid. Revista Iberoamericana de Educación, (65), 91-110. https://www.doi.org/10.35362/rie650395

Sarmiento, J.A., Silva, A.C., y Van Gameren, E. (2019). Evolution of the inequality of educational opportunities from secondary education to university. International Journal of Educational Development, 66, 193-202. https://www.doi.org/10.1016/j.ijedudev.2018.09.006

Tourón, J., Martín, D., Navarro, E., Pradas, S., e Íñigo, V. (2018). Validación de constructo de un instrumento para medir la competencia digital docente de los profesores (CDD). Revista española de pedagogía, 269, 25-54. https://www.doi.org/10.22550/ REP76-1-2018-02

Ugolini, F., Massetti, L., Sanesi, G., y Pearlmutter, D. (2015). Knowledge transfer between stakeholders in the field of urban forestry and green infrastructure: Results of a European sur- 
vey. Land use policy, 49, 365-381. https:/www.doi.org/10.1016/j. landusepol.2015.08.019

Venieris, G., Cohen, S., Vlismas, O., Naoum, V.C., y Karatzimas, S. (2017). The requirements of different user groups on an online accounting platform. Journal for international business and entrepreneurship development, 10(1), 54-70. https://www.doi. org/10.1504/JIBED.2017.082751

Viñals, A., y Cuenca, J. (2016). El rol del docente en la era digital. Revista Interuniversitaria de Formación del Profesorado, 30(2), 103-114.
Wheelahan, L. (2015). Not just skills: What a focus on knowledge means for vocational education. Journal of Curriculum Studies, 47(6), 750-762. https://www.doi.org/10.1080/00220272.2015.1 089942

Widaningsih, L., Barliana, M.S., Aryanti, T., y Malihah, E. (2018). Inheritance pattern of vocational skills: an ethnographic study on construction workers in Indonesia. Journal of technical education and training, 10(2), 71-81. https://www.doi.org/10.30880/ jtet.2018.10.02.007 
